\title{
Rigid and flexible azopolymers modified with donor/acceptor groups. Synthesis and photochromic behavior
}

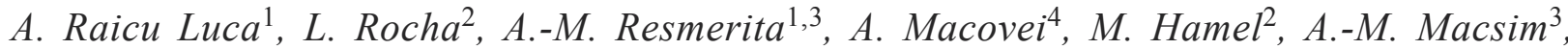 \\ N. Nichita ${ }^{4}$, N. Hurduc ${ }^{*}$ \\ 1'Gheorghe Asachi' Technical University of Iasi, Department of Natural and Synthetic Polymers, Prof. Dimitrie. \\ Mangeron Street, 73, 700050-Iasi, Romania \\ ${ }^{2}$ CEA, LIST Saclay, Laboratoire Capteurs et Architectures Électroniques, F-91191 Gif-sur-Yvette Cedex, France \\ ${ }^{3}$ Institute of Macromolecular Chemistry Petru Poni, Gica Voda Alley 41A, 700487 Iasi, Romania \\ ${ }^{4}$ Institute of Biochemistry of the Romanian Academy, Department of Viral Glycoproteins, Splaiul Independentei 296, \\ Sector 6, Bucharest, Romania
}

Received 16 March 2011; accepted in revised form 18 May 2011

\begin{abstract}
The aim of this work is to investigate the photochromic behavior and nano-structuration capacity of azo-polymers with different architectures and main chain flexibilities, modified with donor/acceptor groups. As a function of the chemical structure and the substitution degree, the azo-polymers can generate physical interactions and lead to different polymer chain conformational re-organization under optical excitation. Nano-structuration experiments were performed on samples with different chemical structures. Surface relief gratings have been realized both in poly(chloromethyl styrene) and polysiloxanes polymers. The complexity of the phenomena that take place under optical excitation of the azo-benzene molecules are reflected by the samples behavior during the nano-structuration process. Preliminary tests to determine the ability of the azo-polysiloxanic films to support cell growth were performed. The films showed remarkable properties to sustain both cell adhesion and proliferation.
\end{abstract}

Keywords: smart polymers, photochromic behavior, cell cultures, SRG, azobenzene

\section{Introduction}

Azobenzene-containing polymers have received increasing attention due to their unique properties allowing various applications, such as in micro-fluidic or in membrane based separation systems, to optically change the materials surfaces wettability [1-5], in biology, to generate photosensitive micellar systems for drug delivery purposes, for example $[6,7]$, in optics or optoelectronics, to generate optical switching and data storage functions [8-13]. The distinct properties of these materials are attributed to the photo-isomerization of azobenzene mol- ecules which undergo reversible trans-cis isomerization of the double bond [14], under external stimuli. It should be emphasized that isomerization of the azobenzene is accompanied by large changes in both geometry and dipole moment [15-19]. These photo responsive properties are determined by both azo-chromophore structure and polymeric architecture.

One of the most interesting applications is connected to the possibility to induce a certain type of relief on the surface of azo-polymeric film, by laser irradiation, [20-22]. This surface nano-structura-

\footnotetext{
${ }^{*}$ Corresponding author, e-mail: nhurduc@ch.tuiasi.ro
}

(c) BME-PT 
tion can be used, for example, to improve the performances of devices such as organic light emitting diodes (OLEDs), organic photovoltaic devices (OPV cells) $[23,24]$ or in biological applications as a support to modify the behavior of cell cultures [25].

It is known that the relief formation is based on a directional flowing process [26]; however, despite the nano-structuration procedure being investigated for more than 15 years $[5,6]$ the mechanism has not been completely elucidated yet. Indeed, the photoinduced mass-transport process, at the base of the surface deformation, enables direct production of surface relief gratings, through exposition of the films to a spatially modulated intensity pattern. This method appears thus as a simple tool enabling the realization of complex surface structures, through a single step procedure [27]. An important aspect concerning the relief quality is the geometrical surface stability, directly connected with the glass transition temperature $\left(T_{\mathrm{g}}\right)$ value of the polymer. Especially in the case of polysiloxane main-chain systems, some problems concerning the surface relief stability can appear due to $T_{\mathrm{g}}$ values close to room temperature. While the high flexibility of the polysiloxane backbone should provide an increased sensitivity of the azopolymer film topography to light stimulation, a competition between the optically controlled mass transport and the isotropic thermal redistribution of the material at ambient temperature can occur. This phenomenon will then limit the potential of such materials for applications involving nanostructured films. In this case, the physical interactions between donor/acceptor (D/A) groups connected to the polymeric chains can offer a supplementary stability of the surface relief.

In this context, the aim of this work was to investigate the photochromic behavior and nano-structuration capacity of azo-polymers with different architectures and main chain flexibilities, modified with D/A groups. Two types of polymer architectures were investigated: flexible main-chain polysiloxanes and rigid poly(chloromethyl styrene) derivatives. Herein, we studied the photo-isomerization process in solution and solid states, and the effect of polymers backbone and substitution degree with azobenzene and D/A groups in these processes. Preliminary tests concerning the nano-structuration capacity were also performed at room temperature, using the intensity modulations of an interference pattern produced with laser irradiation [28]. The interest to obtain azo-polysiloxane nano-structured films is moreover justified by their potential biological applications. Thus, preliminary cell proliferation experiments using two different cell lines evidenced not only a normal attachment to the substrate, but also, a division rate similar to controls, even on the plane films. This behavior is very encouraging, especially in the light of the literature data published so far in the field. To our knowledge, there is only one study reporting the possibility to use azopolymer films as cell growth support. This showed that a commercial copolymer poly[(methylmethacrylate)-co-(disperse red 1 acrylate)], was not able to sustain the development of fibroblast cells on the plane surface of the azopolymeric films, only the structured region allowing cell adhesion and growth [25].

\section{Experimental part}

Methylene chloride (code 270997), dimethylsulfoxide (code 494429), methanol (code 494437), chloroform (code 472476) 4-phenylazophenol (code 131083) and 2,4-dinitrophenol (code 42170) were purchased from Aldrich, Steinheim, Germany and used without supplementary purification. The ((chloromethyl)phenylethyl)methyldichlorosillane (AB110958) was purchased from ABCR GmbH \& Co. KG, Karlsruhe, Germany and used without supplementary purification. The azopolymers were synthesized in two steps by nucleophilic substitution of chlorobenzyl groups with the sodium salt of 4-(phenylazo) phenol or 2,4-dinitrophenol as shown in Figure 1. More details concerning polymer synthesis can be found in our previous reports $[29,30]$. All polymers were characterized by ${ }^{1} \mathrm{H}-\mathrm{NMR}$, differential scanning calorimetry (DSC), UV spectroscopy and surface contact angle measurements. The photochromic behavior (azobenzene trans-cis isomerization and the cis-trans relaxation phenomena) was investigated in solid state by UV spectroscopy using a Shimadzu spectrophotometer (Shimadzu UV 1700 Pharma Spy, Kyoto, Japan). For the measurements in solid state, thin film were coated on the surface of a quartz cuvette. The azopolymer solutions and films were irradiated using a UV lamp (100 W) equipped with $365 \mathrm{~nm}$ filter and during irradiation experiments the surface of the films was cooled with compressed air, the tempera- 


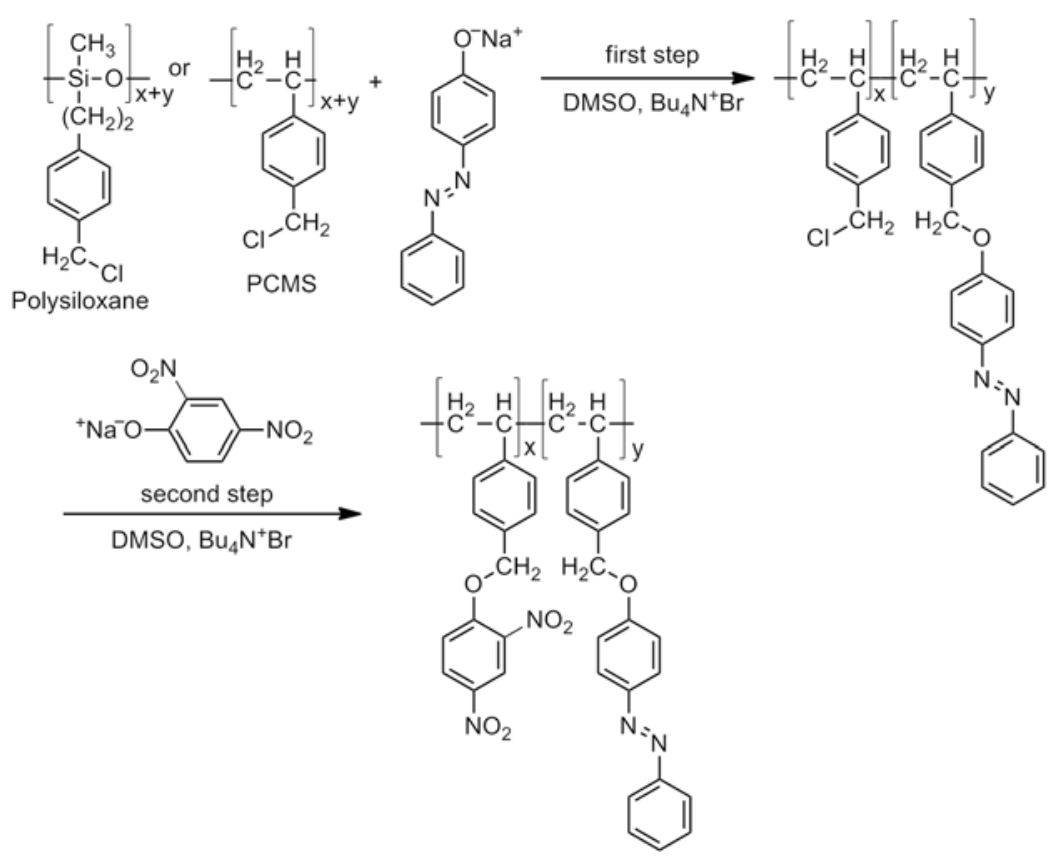

Figure 1. Synthesis of azopolymers modified with 2,4-dinitrophenol

ture being maintained at $22^{\circ} \mathrm{C}\left( \pm 1^{\circ} \mathrm{C}\right)$. The isomerization kinetics of azobenzene can be investigated due to the fact that the trans and the cis isomers absorption occurs at different wavelengths: a strong absorption peaking at $350 \mathrm{~nm}$, attributed to the $\pi-\pi^{*}$ of azobenzene chromophore and a weak broad band in the visible region with a maximum at $440 \mathrm{~nm}$, corresponding to the $n-\pi *$ electronic transition of the same moiety. During UV irradiation at $365 \mathrm{~nm}$ the absorbance corresponding to the $\pi-\pi^{*}$ transition of azobenzene strongly decreases, whereas intensity of the $n-\pi^{*}$ electronic transition slightly increases. Subsequently, visible light irradiation leads to recovery of the shape of the absorbance spectra, but only partially. ${ }^{1} \mathrm{H}-\mathrm{NMR}$ spectra were recorded on Bruker $400 \mathrm{MHz}$ apparatus (Bruker, Rheinstetten, Germany). DSC analysis was performed with a Mettler DSC-1 machine (METTLER-TOLEDO GMB, Switzerland) with a heating rate $10^{\circ} \mathrm{C} / \mathrm{min}$ under $\mathrm{N}_{2}$ atmosphere. The GPC (Polymer Laboratories PLGPC 129 United Kingdom) measurements were effectuated in THF using polystyrene standard. The isomerization phenomena were measured with the Shimadzu spectrophotometer. The static contact angle values were measured using EasyDrop Shape Analysis System (KRUSS Easy Drop Standard, Hamburg, Germany) equipped with DSA 1 evaluation software. A drop of liquid (10 $\mu \mathrm{l})$ is placed on the film surface.
The nanostructured surfaces were obtained by illumination of the polymer films with an interference pattern produced by the superposition of two coherent light beams absorbed by the azo-molecules. The beams were incident with a $\theta$ angle on the film surface, schematically show in Figure 2, resulting in a pattern with a sinusoidal modulated intensity. The excitation was performed in the visible range, with a laser diode delivering a $488 \mathrm{~nm}$ wavelength beam. The intensity of irradiation was $170 \mathrm{~mW} / \mathrm{cm}^{2}$ and the beams were p-polarized, i.e. with a polarization in the beams incidence plane. In this configuration the polarization axes are perpendicular to the fringes of the interference pattern, in order to favor a migration of the material from the high intensity regions of the interference pattern to the low ones [31].

The HepG2 (human hepatoblastoma) and HeLa (human ovarian cancer) cells were maintained at $37^{\circ} \mathrm{C}$, in RPMI medium supplemented with $10 \%$ fetal calf serum, 50 units $/ \mathrm{ml}$ penicillin, $50 \mu \mathrm{g} / \mathrm{ml}$ streptomycin and 2 mM GlutaMAX (Gibco ${ }^{\circledR}$ Cell Culture,

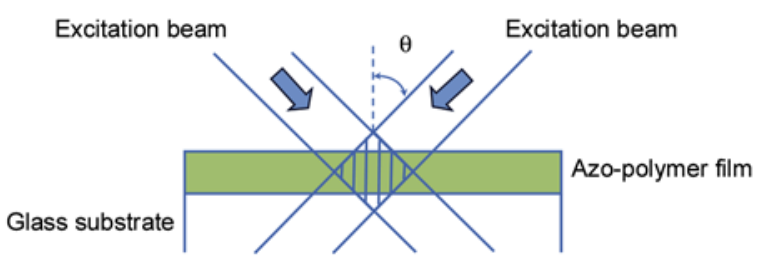

Figure 2. Excitation configuration for the induction of surface relief gratings in azo-polymer films 
USA) in a $5 \% \mathrm{CO}_{2}$ atmosphere. An equal number of cells were seeded onto the azo-polymer film and control (microscope cover glass) substrates. Cell attachment and multiplication were visualized 24 hours later, using an inverted Nikon microscope (Nikon Instruments Europe B.V.)

\section{Results and discussion}

Two different polymer groups (having a flexible or a rigid main-chain) modified with 4-(phenyl-azo) phenol and 2,4-dinitrophenol were investigated, in order to identify the best candidates concerning the nano-structuration process. Some characteristics of the synthesized polymers are presented in Table 1.

The synthesized polymers were modified with azobenzene and 2,4-dinitrophenol, to a 45-90 and $22-56 \%$ substitution degree, respectively, The molecular weights $\left(M_{\mathrm{n}}\right)$ of the polymers are situated in the range of 5900 to 8800 . The glass transitions $T_{\mathrm{g}}$ values are strongly influenced by the main-chain flexibility being located between 15 and $30^{\circ} \mathrm{C}$, in the case of polysiloxanes and from 106 to $123^{\circ} \mathrm{C}$, in the case of modified PCMS.

The UV-Vis spectra (in solid state) corresponding to the synthesized polymers are presented in Figure 3. All the films, except sample 9, are characterized by two peaks of absorbance. The weak broad peak, in the visible region at $440 \mathrm{~nm}$, corresponds to both the trans and the cis isomers and the strong peak, with maximum at $343-345 \mathrm{~nm}$ is attributed to the trans isomer absorption. These two configurations can be easily inter-converted by light, involving large changes in dipole moment and geometry. When the films are exposed to UV light, the stable trans form (lower energy) can be photo-isomerized

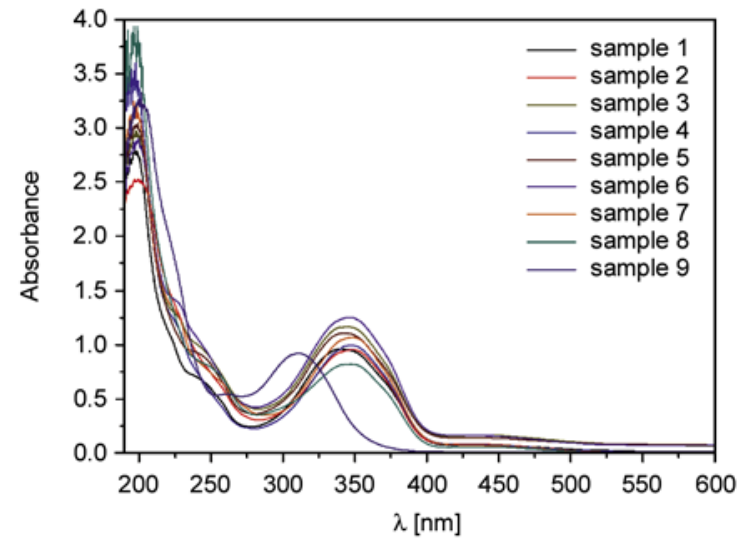

Figure 3. UV-Vis spectra in solid state of azo-polymers

to the methastable cis form (higher energy), the cistrans relaxation process is occurring thermally or/and photo-chemically (induced by visible light). In the case of samples 7 and 8 a red-shift displacement of the maximum absorption corresponding to the trans-azobenzene isomer can be observed, suggesting some association processes between azoand 2,4-dinitrophenol groups.

Upon irradiation of the film at $365 \mathrm{~nm}$, the strong absorption band $(345 \mathrm{~nm})$ decreases in intensity, reflecting the isomerization process and the cis-isomer formation. Based on this absorption band at $345 \mathrm{~nm}$, the percentage of the cis-isomer can be calculated and the kinetic curves of the isomerization process can be plotted. In Figure 4, for similar substitution degrees of the polysiloxane and the poly (chloromethyl styrene), the main-chain flexibility influences significantly the photochromic behavior only if the substitution degree is lower than 50\% (samples 2 and 4). In the other cases, only the chemical structure of the substituents influences the maximum conversion degree in cis-isomer. For

Table 1. Characteristics of the synthesized polymers

\begin{tabular}{|c|c|c|c|c|c|}
\hline Sample no. & Polymer & Substituents & Substitution degree [\%] & $\mathbf{M}_{\mathbf{n}}$ & $\mathbf{T}_{\mathrm{g}}\left[{ }^{\circ} \mathbf{C}\right]$ \\
\hline 1 & Polysiloxanes & Azobenzene & 90 & 7500 & 30 \\
\hline 2 & Polysiloxanes & Azobenzene & 46 & 5900 & 15 \\
\hline 3 & PCMS & Azobenzene & 82 & 7320 & 115 \\
\hline 4 & PCMS & Azobenzene & 45 & 8800 & 112 \\
\hline 5 & Polysiloxanes & $\begin{array}{l}\text { Azobenzene } \\
\text { 2,4-dinitrophenol }\end{array}$ & $\begin{array}{l}73 \\
22\end{array}$ & 8000 & 29 \\
\hline 6 & PCMS & \begin{tabular}{|l|} 
azobenzene \\
2,4-dinitrophenol
\end{tabular} & $\begin{array}{l}60 \\
23\end{array}$ & 6800 & 123 \\
\hline 7 & Polysiloxanes & $\begin{array}{l}\text { Azobenzene } \\
\text { 2,4-dinitrophenol }\end{array}$ & $\begin{array}{l}50 \\
24\end{array}$ & 7500 & 23 \\
\hline 8 & PCMS & $\begin{array}{l}\text { Azobenzene } \\
\text { 2,4-dinitrophenol }\end{array}$ & $\begin{array}{l}51 \\
26\end{array}$ & 7000 & 112 \\
\hline 9 & Polysiloxanes & 2,4-dinitrophenol & 56 & 5900 & - \\
\hline
\end{tabular}




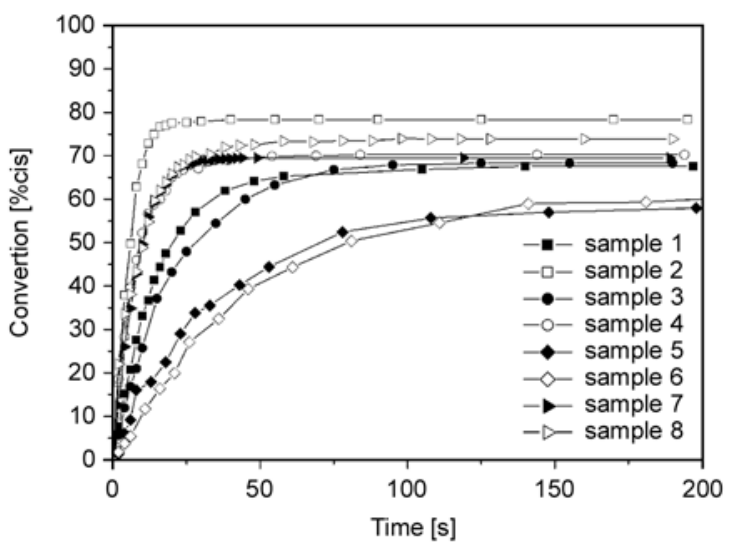

Figure 4. The kinetic curves corresponding to the trans-cis isomerization in solid state of the synthesized azo-polymerst

materials with 2,4-dinitrophenol, a strong difference concerning the maximum cis-isomer content and the time constant to reach the plateau value was observed when the azobenzene groups content decreased (samples 5 and 6 compared to samples 7 and 8). An increase of the maximum content of azobenzene molecules in the cis form also occurs for the polymers substituted with azobenzene molecules only, when the substitution degree is reduced. However, the isomerization kinetic modifications are smaller for high substitution degrees. For example, the maximum content of azobenzene molecules in the cis form can be $11 \%$ higher for sample 2 compared to sample 1 while the substitution degree of azobenzene molecules in sample 2 is almost the half of sample 1. Comparison of samples 5 and 7 , shows that for equivalent 2,4-dinitrophenol substitution degrees, a similar increase (12\%) of the maximum content of azobenzene molecules in the cis

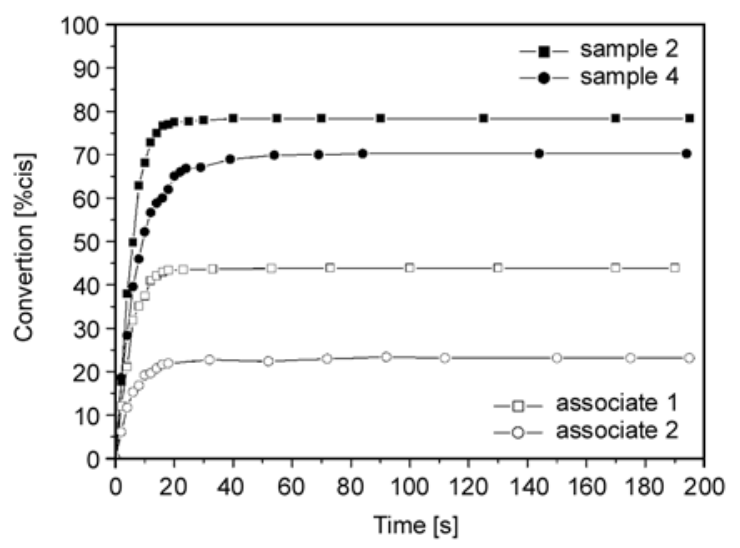

Figure 5. The kinetic curves corresponding to the trans-cis isomerization process (in solid state) of the associate 1 between sample 2 and sample 9, associate 2 between sample 4 and sample 9 . form results from only a $23 \%$ decrease of the azobenzene substitution degree. This suggests some association processes between 2,4-dinitrophenol and azobenzene groups, in agreement with UV-VIS spectral information. To verify this assumption, an associate between samples 2 and 9 (gravimetric ratio 1:1) was prepared, dissolving both polymers in chloroform and evaporating the solvent. Figure 5 shows the difference concerning the photochromic behavior of this associate compared to sample 2. In the case of the associate, the maximum cis-isomer content is 44 , by $32 \%$ lower than that of sample 2 .

Sample 9 (a polymer substituted only with 2,4-dinitrophenol) was preferred to verify the association capacity of the azo-polymers, taking into consideration the potential steric hindrance generated by the length differences between azobenzene and 2,4dinitrophenol groups.

The associations between azo and 2,4-dinitrophenol groups are more evident during the cis-trans relaxation processes (Figure 6 and Figure 7). Moreover, the association influences are more evident in the case of the thermal relaxations which take place much slower compared with the visible light induced one. For the visible light induced relaxation in Figure 6, a step-way process was also observed for samples 5 and 6 .

This step-way relaxation is very clearly reflected when the cis-trans isomerization is activated only thermally (Figure 6, samples 5 and 6). In the case of samples 7 and 8 , the relaxation rate is strongly diminished by the interactions between 2,4-dinitrophenol and azo-groups. Thus, more than 12 and 10 days, respectively, were necessary for samples 7 and 8 to obtain a $100 \%$ trans-isomer. The samples behavior during relaxation suggests a complicated

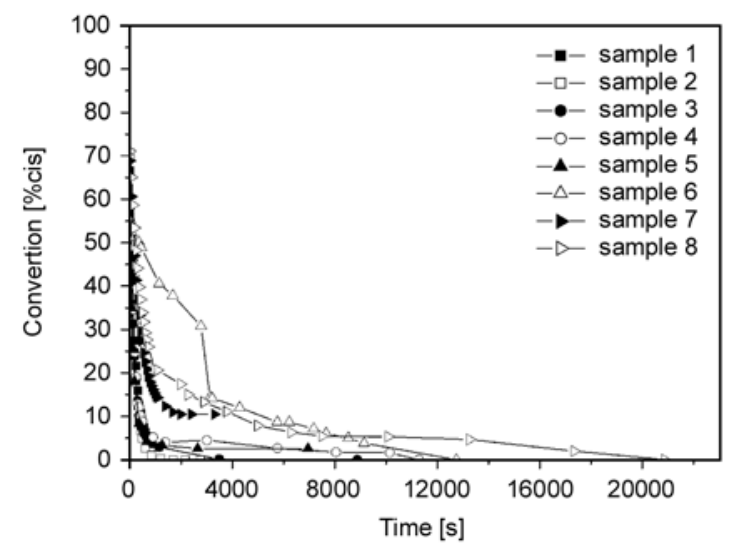

Figure 6. The kinetic curves corresponding to the cis-trans relaxation in visible light of azo-polymers 


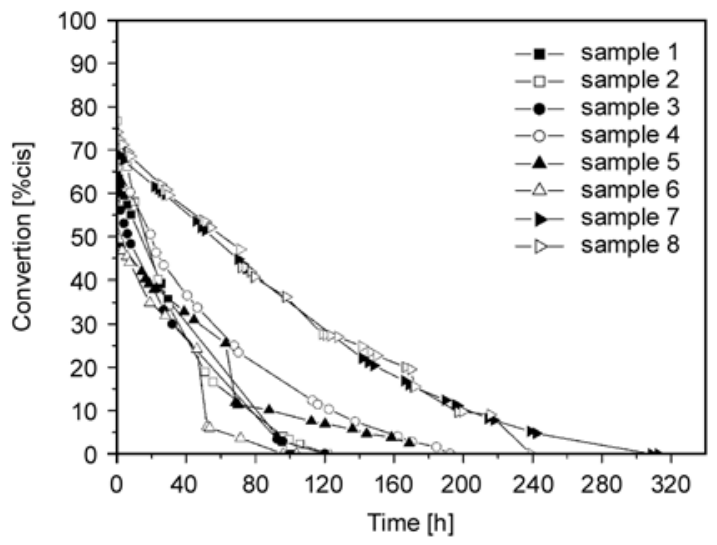

Figure 7. The kinetic curves corresponding to the cis-trans thermal relaxation of azo-polymers

chain re-ordering process that imposes supplementary studies.

The complexity of the re-ordering processes was also confirmed by the contact angle measurement. During the azobenzene isomerization, the wettability of the surface was also modified, as a result of geometry and dipole moment changes. Previous molecular modeling studies [32] evidenced a dipolemoment value for the trans-azophenol of 1.6 D that increases to $4.5 \mathrm{D}$ in the cis configuration. These molecular modeling studies showed the displacement of the azo-groups at the film surface. In addition, we carried out contact angle experiments for the non-irradiated polymeric film and during the relaxation process (Figure 8).

The contact angle values of the non-irradiated sample are placed at $t=0 \mathrm{~min}$, the first point after irradiation corresponding to the value $t=2 \mathrm{~min}$. After 2 minutes of irradiation, important modifications of the surface properties take place as a consequence

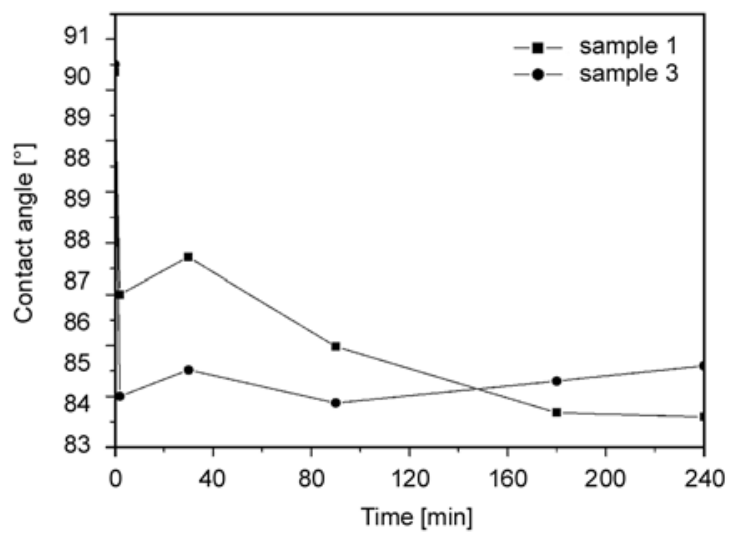

Figure 8. Evolution of contact angle over time for the azopolysiloxanes and azo-PCMS in dark; non-radiated, 30, 90, 180 and 240 min after irradiation, solvent water (Samples 1 and 3 - Table 1) of the trans-cis isomerization process of the azogroups.

Another conclusion is connected to the chains reorganization processes; this is more evident in the case of the flexible main-chain polymer, the contact angle value continuously decreasing during the 30 240 min interval, after irradiation. In the case of the rigid main-chain (PCMS) the changing of the surface properties is only the consequence of the dipole-moment increasing from 1.6 to $4.5 \mathrm{D}$, due to the trans-cis isomerization process.

Preliminary studies concerning the nano-structuration capacity under visible laser irradiation $(\lambda=$ $488 \mathrm{~nm}$ ) were performed. The typical modulation dynamics representing the evolution of the modulation amplitudes in function of the irradiation time is shown in Figure 9. The curve is obtained by recording with a photodiode the diffracted intensity of a He-Ne laser beam sent onto the irradiated area of the polymer film, during the irradiation as a probe. The $633 \mathrm{~nm}$ wavelength of the He-Ne laser was chosen since it is not absorbed by the azobenzene molecules and thus would not perturb the film structuration process.

The curve presented in Figure 9 shows a sigmoidal shape, with a slow increase in the first minutes followed by a rapid increase, reaching finally a plateau value. The slow increase in the first minutes can be related to a periodical re-organization of the azobenzene molecules occurring in the bulk, while for longer irradiation times the increase of the dif-

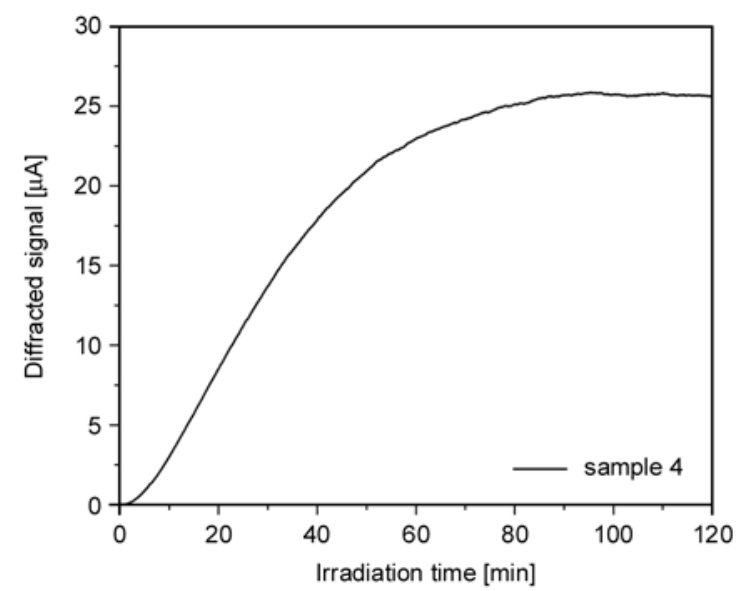

Figure 9. Evolution of the He-Ne laser beam intensity diffracted by a polymer film of sample 4 exposed to an interference pattern in function of the irradiation time. The curve is related to the evolution of the modulation amplitudes recorded in film surface during the irradiation. 


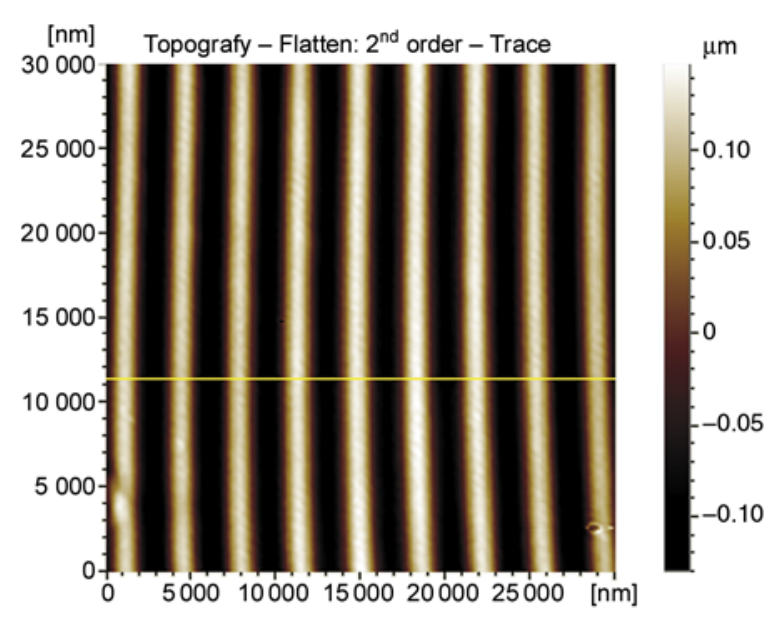

a)

Figure 10. AFM topographic image (a) and cross section (b) of a surface grating induced in a thin film of sample 4 after laser irradiation in the visible range $(\lambda=488 \mathrm{~nm})$

fracted intensity has been correlated to an increase of the modulation amplitudes.

When the signal reached the plateau value, the experiment was stopped and the topography of the irradiated surface was characterized by atomic force microscopy (AFM). The next figure shows an image of the surface topography of an irradiated area of the film.

Figure 10 shows a sinusoidal modulation of the film surface, as a result of the excitation, with the interference pattern presenting a sinusoidal modulated intensity profile. The period corresponds to the fringes spacing of the interference pattern, which is set by the $\theta$ incidence angle. In the present case gratings with a period of $\Lambda=3390 \mathrm{~nm}$ and a modulation amplitude of $\Delta h=235 \mathrm{~nm}$ were obtained.

The sample with similar azo-benzene content (46\%) but having a flexible polymeric main-chain (sample 2) was irradiated under the experimental conditions described above. The He-Ne diffracted intensity-time curve in Figure 11 shows a small increase of the diffracted signal intensity (three orders of magnitude lower than that of the PCMS) limited to the first minutes before a stable signal intensity is reached. AFM measurements confirmed that any surface grating has been inscribed in sample 2. An explanation lays probably in the very low $T_{\mathrm{g}}$ value of this material $\left(T_{\mathrm{g}}=15^{\circ} \mathrm{C}\right)$, which does not allow sufficient stability onto the polymer film, the induced surface deformations being continuously erased at ambient temperature, during the irradiation.
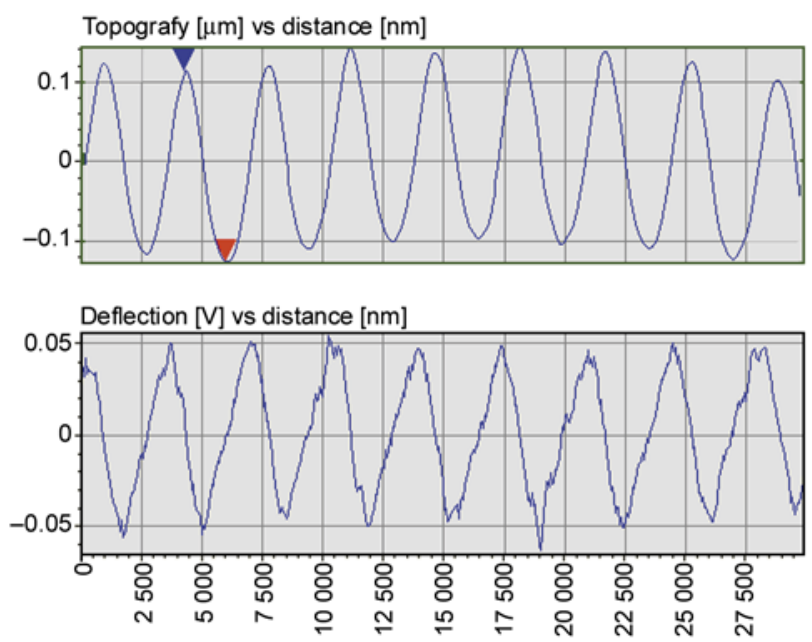

b)

This hypothesis was confirmed by experiments performed on sample 7, with an azobenzene substitution degree close to that of sample 2, but with additional 2,4-dinitrophenol groups (substitution degree $24 \%$ ). In this case, the material has a $T_{\mathrm{g}}$ close to $23^{\circ} \mathrm{C}$, higher than the $T_{\mathrm{g}}$ of sample 2 . For this material, we were able to record a variation of the diffracted $\mathrm{He}-\mathrm{Ne}$ intensity with a similar shape to that of sample 4. The result is illustrated in Figure 12 for both samples and normalized intensities.

The evolution of the diffracted intensities shows a sigmoidal shape for both samples, however, it is important to note that a significantly slower evolution of the intensity is observed for sample 7. Indeed, the saturation is reached after 70 minutes for sample 4, while for sample 7 almost 2 hours are needed.

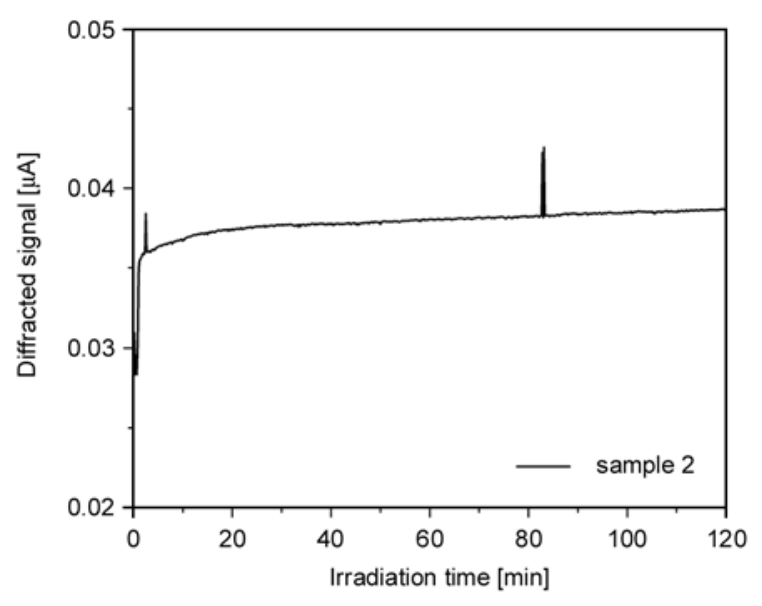

Figure 11. Plot of the He-Ne laser intensity diffracted by the film as a function of irradiation time 


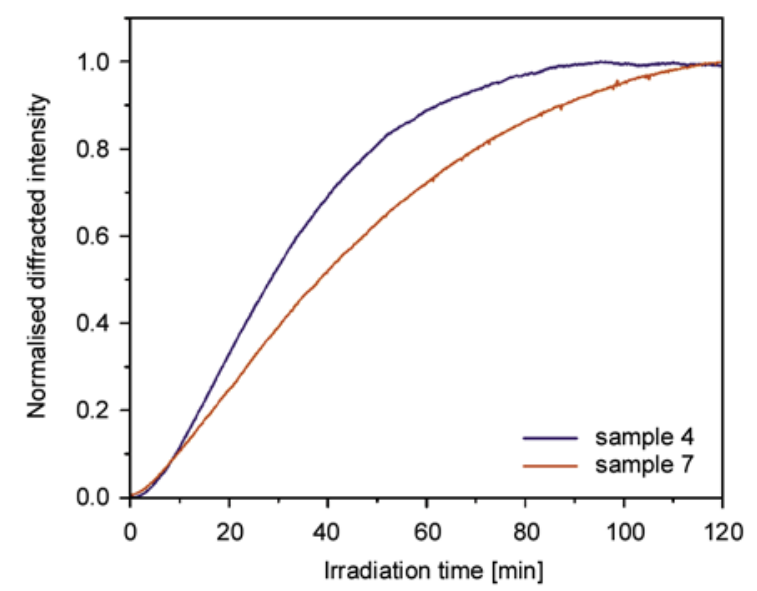

Figure 12. Normalized diffracted He-Ne laser beam intensity in function of the irradiation time for sample 4 and sample 7

A first explanation for this behavior may rely on the polymer chain properties. The different flexibilities of the poly(chloromethyl styrene) and the polysiloxane matrices resulting in different $T_{\mathrm{g}}$ can indeed have strong consequences on the stability of the surface relief grating formation and thus on the efficiency of the process, as highlighted by sample 2 . Another possible explanation concerns an effect of association between the 2,4-dinitrophenol and the azobenzene molecules on the trans-cis-trans isomerization of the azobenzene, which is the driving force in the nano-structuration process. Indeed, while a direct influence of the 2,4-dinitrophenol additional groups on the azobenzene molecules isomerization is not visible when comparing samples 4 and 7; since these materials present similar trans to cis conversion kinetics and efficiencies (Figure 4), the photo-induced relaxation appears to be less efficient for sample 7, as evidenced in Figure 5. A reduction of the isomerization complete cycle efficiency is coherent with a slower modulation dynamics for sample 7. As well, the influence of the 2,4-dinitrophenol groups on the flexibility and the conformational reorganization process of the polysiloxane chains around the azobenzene molecules could play an important part in the structuration process. A competition between physical associations occurring in the azo-polymers containing donor/acceptor groups (as evidenced by Figures 4 and 5) and the polymer main chain flexibility is thus expected to take place. The precise identification of the parameters influencing the surface structure is not an easy task and will need further experiments involving azo-polymers with different donor/acceptor groups and substitution degrees, in order to selectively modify each feature of the materials. Additionally, the investigation of the relative contributions of the birefringence grating, resulting from the photoinduced azobenzene molecules angular redistribution, and the surface grating can be an efficient tool in understanding the influence of the material chemical structure on the nano-structuration mechanisms [33-35].

Preliminary tests to determine the ability of the azopolysiloxanic films to support cell growth were also performed using the HepG2 and HeLa cell lines, of human hepatic and epithelial origin. The films showed remarkable properties to sustain both cell adhesion and proliferation, as revealed by microscopy analysis of the cell cultures at 24 hours postseeding (Figure 13). Importantly, the films were easily sterilized by incubation in 100\% ethanol, for 30 minutes and were stable during all cell culture procedures. These promising results make the azopolymer films important candidates for future biological applications.

\section{Conclusions}

The possibility to obtain azo-polymers with flexible or rigid main-chain, containing $\mathrm{D} / \mathrm{A}$ group was presented. The chemical structure, photochromic behavior and surface nano-structuration capacity of the polymers were characterized. Depending on the chemical structure and the substitution degree, the azo-polymers can generate physical interactions and modify the materials conformational re-organization, after optical excitation, with important consequences on the nano-structure of the azo-polymer films. These associations were evidenced by UV/ VIS spectroscopy or by a specific photochromic behavior, especially during the cis-trans relaxation processes. The complex reorganization process after the UV irradiation, especially in the case of flexible main-chain polymers, was evidenced by the photochromic behavior study related to the contact angle measurements. The complex phenomena that take place during the nano-structuration process were reflected in the behavior of three samples during laser irradiation. A significantly faster modulation was observed in the case of PCMS rigid mainchain, suggesting that interactions between azobenzene and dinitro-phenol groups coupled to 


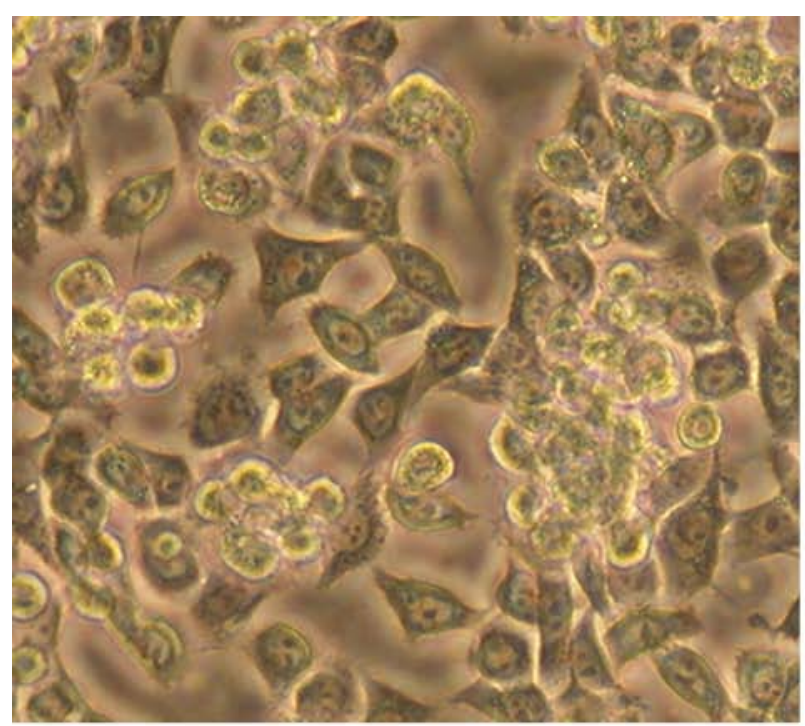

a)

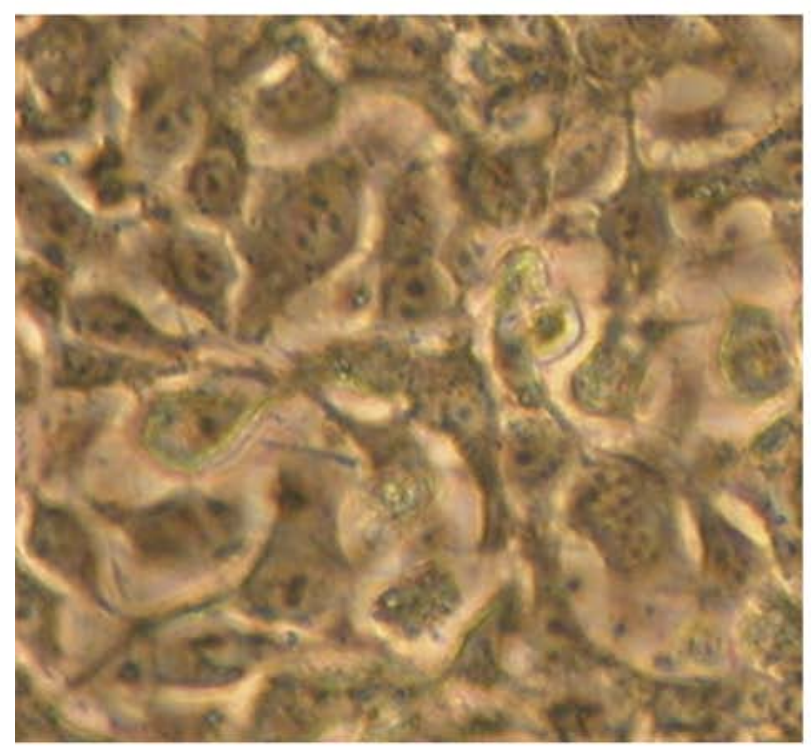

c)

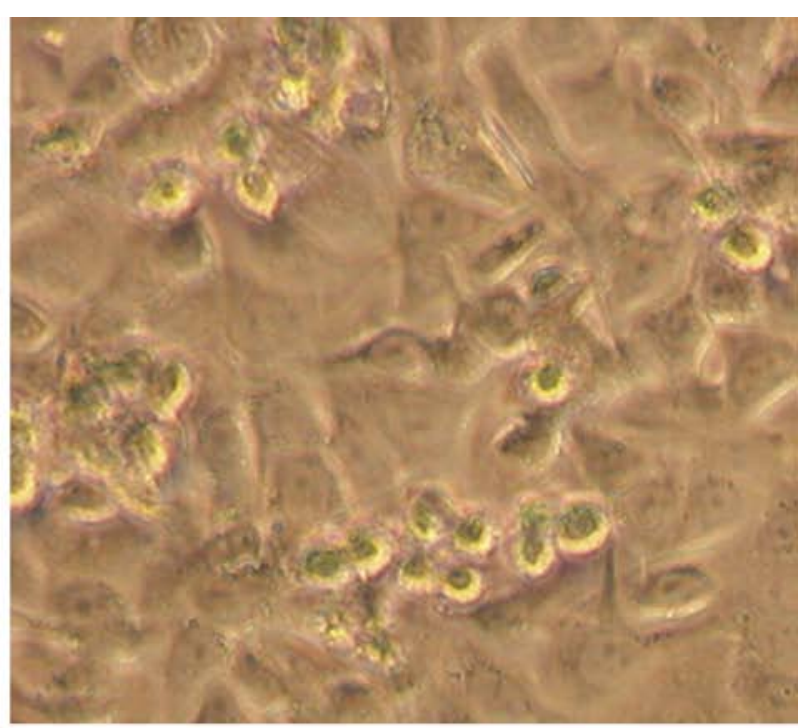

b)

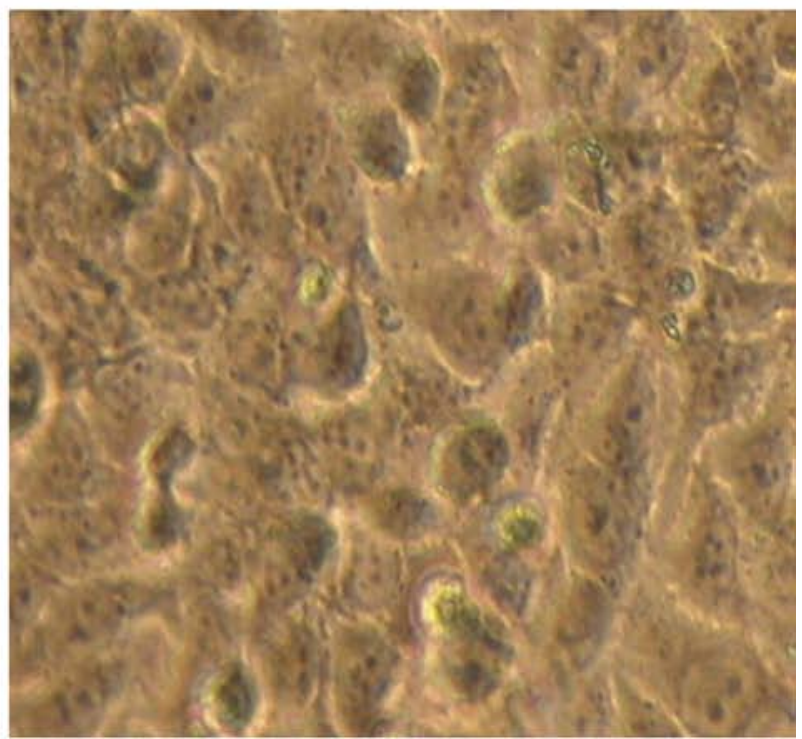

d)

Figure 13. Equal number of HepG2 (a, b) and HeLa (c, d) cells were seeded on either azo-polymer (Sample 1) film (a, c) or microscope cover glass, as control (b, d). Cell development was visualized at 24 hours post-seeding, using an inverted microscope (20× magnification).

supplementary re-ordering phenomena permitted by the chain flexibility in polysiloxane based azopolymers can play an important role in the differences observed on the relief formation processes. The first results reported on the nano-structuration properties are promising, as concerns the use of additional donor/acceptor groups to control the structuration properties of flexible azo-polysiloxanes films. These preliminary experiments represent a first step towards the development of materials with high sensitivity to optical stimulation, due to a flexible polymer backbone, and efficient and stable structure properties, at the same time. A better understanding of the phenomena involved azo-polysiloxanes will require further optimization studies regarding the nano-structuration properties of such polymer films. Preliminary tests to determine the ability of the azo-polysiloxanic films to support cell growth were performed. The films showed remarkable properties to sustain both cell adhesion and proliferation. These promising results make the azo-polymer films important candidates for future biological applications. 


\section{Acknowledgements}

The Romanian authors want to thank to ANCS for financial support of this research (Grant CEA - C1- 01/2010) and to project BRAIN "Doctoral scholarships as an investment in intelligence", financed by the European Social Found and Romanian Government

\section{References}

[1] Feng C. L., Zhang Y. J., Jin J., Song Y. L., Xie L. Y., Qu G. R., Jiang L., Zhu D. B.: Reversible wettability of photoresponsive fluorine-containing azobenzene polymer in langmuir-blodgett films. Langmuir, 17, 4593-4597 (2001).

DOI: $10.1021 / 1 \mathrm{a} 010071 \mathrm{r}$

[2] Jiang W., Wang G., He Y., Wang X., An Y., Song Y., Jiang L.: Photo-switched wettability on an electrostatic self-assembly azobenzene monolayer. Chemical Communications, 2005, 3550-3552 (2005).

DOI: $10.1039 / \mathrm{B} 504479 \mathrm{~K}$

[3] Lim H. S., Han J. T., Kwak D., Jin M., Cho K.: Photoreversibly switchable superhydrophobic surface with erasable and rewritable pattern. Journal of the American Chemical Society, 128, 14458-14459 (2006). DOI: $10.1021 / \mathrm{ja} 0655901$

[4] Cao H. Z., Zhang W., Zhu J., Chen X. R., Cheng Z. P., Wu J. H., Zhu X. L.: Azo polymers with electronical push and pull structures prepared via RAFT polymerization and its photoinduced birefringence behavior. Express Polymer Letters, 2, 589-601 (2008).

DOI: $10.3144 /$ expresspolymlett.2008.71

[5] Zhao X. Y., Wang M. Z.: Structure dependence of photochromism and thermochromism of azobenzene-functionalized polythiophenes. Express Polymer Letters, 1, 450-455 (2007).

DOI: $10.3144 /$ expresspolymlett.2007.63

[6] Wang G., Tong X., Zhao Y.: Preparation of azobenzene-containing amphiphilic diblock copolymers for light-responsive micellar aggregates. Macromolecules, 37, 8911-8917 (2004).

DOI: $10.1021 / \mathrm{ma} 048416 \mathrm{a}$

[7] Moleavin I., Grama S., Cârlescu I., Scutaru D., Hurduc N.: Photosensitive micelles based on polysiloxanes containing azobenzene moieties. Polymer Bulletin, 65, 69-81 (2010).

DOI: $10.1007 / \mathrm{s} 00289-010-0247-4$

[8] Jiang X., Wang H., Chen X., Li X., Lei L., Mu J., Wang G., Zhang S.: A novel photoactive hyperbranched poly(aryl ether ketone) with azobenzene end groups for optical storage applications. Reactive and Functional Polymers, 70, 699-705 (2010). DOI: $10.1016 /$ j.reactfunctpolym.2010.06.005

[9] Natansohn A., Rochon P.: Photoinduced motions in azocontaining polymers. Chemical Reviews, 102, 41394175 (2002). DOI: $10.1021 / \mathrm{cr} 970155 \mathrm{y}$
[10] Pan X., Wang C., Xu H., Wang C., Zhang X.: Polarization holographic gratings in an azobenzene side-chain liquid-crystalline polymer. Applied Physics B: Lasers and Optics, 86, 693-697 (2007). DOI: $10.1007 / \mathrm{s} 00340-006-2482-9$

[11] Wu S., Yao S., She W., Luo D., Wang H.: All-optical switching properties of poly(methyl methyacrylate) azobenzene composites. Journal of Materials Science, 38, 401-405 (2003).

DOI: $10.1023 / \mathrm{A}: 1021878710507$

[12] Ikeda T., Tsutsumi O.: Optical switching and image storage by means of azobenzene liquid-crystal films. Science, 268, 1873-1875 (1995).

DOI: $10.1126 /$ science. 268.5219 .1873

[13] Berg R. H., Hvilsted S., Ramanujam P. S.: Peptide oligomers for holographic data storage. Nature, 383, 505-508 (1996).

DOI: $10.1038 / 383505 \mathrm{a} 0$

[14] Dürr H., Bouas-Laurent H.: Photochomism: Molecules and systems. Elsevier, Amsterdam (2003).

[15] Xue X., Zhu J., Zhang Z., Zhou N., Zhu X.: Synthesis and photoresponsive behavior of the high- $T_{\mathrm{g}}$ azobenzene polymers via RAFT polymerization. Reactive and Functional Polymers, 70, 456-462 (2010). DOI: $10.1016 /$ j.reactfunctpolym.2010.04.006

[16] Pei S., Chen X., Wang F., Jiang Z., Peng W.: Dynamics of the photo-induced orientation and relaxation of a novel hyperbranched poly(aryl ether) containing azobenzene groups. Laser Physics, 20, 1144-1148 (2010). DOI: 10.1134/S1054660X1009015X

[17] Sun M., Que W., Hu X.: Preparation and trans-cis isomerization of azobenzene-containing $\mathrm{TiO}_{2} /$ ormosils hybrid films derived at a low temperature sol-gel process for photonic applications. Journal of Sol-Gel Science and Technology, 50, 415-420 (2009). DOI: $10.1007 /$ s 10971-009-1918-1

[18] Bobrovsky A., Shibaev V., Hamplova V., Kaspar M., Glogarova M.: Chirooptical and photooptical properties of a novel side-chain azobenzene-containing LC polymer. Monatshefte für Chemie, 140, 789-799 (2009).

DOI: $10.1007 / \mathrm{s} 00706-009-0108-8$

[19] Kumar G. S., Neckers D. C.: Photochemistry of azobenzene-containing polymers. Chemical Reviews, 89, 1915-1925 (1989).

DOI: $10.1021 / \mathrm{cr} 00098 \mathrm{a} 012$

[20] Rochon P., Batalla E., Natansohn A.: Optically induced surface gratings on azoaromatic polymer films. Applied Physics Letters, 66, 136-138 (1995). DOI: $10.1063 / 1.113541$

[21] Kim D. Y., Tripathy S. K., Li L., Kumar J.: Laserinduced holographic surface relief gratings on nonlinear optical polymer films. Applied Physics Letters, 66, 1166-1168 (1995). DOI: $10.1063 / 1.113845$ 
[22] Sobolewska A., Miniewicz A., Grabiec E., Sek D.: Holographic grating recording in azobenzene functionalized polymers. Central European Journal of Chemistry, 4, 266-284 (2006).

DOI: $10.2478 / \mathrm{s} 11532-006-0003-7$

[23] Hubert C., Fiorini-Debuisschert C., Hassiaoui I., Rocha L., Raimond P., Nunzi J-M.: Emission properties of an organic light-emitting diode patterned by a photoinduced autostructuration process. Applied Physics Letters, 87, 191105-191107 (2005).

DOI: $10.1063 / 1.2126793$

[24] Cocoyer C., Rocha L., Sicot L., Geffroy B., de Bettignies R., Sentein C., Fiorini-Debuisschert C., Raimond P.: Implementation of submicrometric periodic surface structures toward improvement of organic-solar-cell performances. Applied Physics Letters, 88, 133108133110 (2006).

DOI: $10.1063 / 1.2188600$

[25] Choi C-H., Heydarkhan-Hagvall S., Wu B. M., Dunn J. C. Y., Beygui R. E., Kim C-J.: Cell growth as a sheet on three-dimensional sharp-tip nanostructures. Journal of Biomedical Materials Research Part A, 89, 804-817 (2009). DOI: $10.1002 / \mathrm{jbm} . \mathrm{a} .32101$

[26] Karageorgiev P., Neher D., Schulz B., Stiller B., Pietsch U., Giersig M., Brehmer L.: From anisotropic photo-fluidity towards nanomanipulation in the optical near-field. Nature Materials, 4, 699-703 (2005). DOI: $10.1038 /$ nmat1459

[27] Hubert C., Malcor E., Maurin I., Nunzi J-M., Raimond P., Fiorini C.: Microstructuring of polymers using a light-controlled molecular migration processes. Applied Surface Science, 186, 29-33 (2002). DOI: $10.1016 / \mathrm{S} 0169-4332(01) 00658-4$

[28] Rocha L., Dumarcher V., Malcor E., Fiorini C., Denis C., Raimond P., Geffroy B., Nunzi J-M.: Photo-induced microstructured polymers for the optimisation and control of organic devices emission properties. Synthetic Metals, 127, 75-79 (2002). DOI: $10.1016 / \mathrm{S} 0379-6779(01) 00599-9$
[29] Hurduc N., Enea R., Scutaru D., Sacarescu L., Donose B. C., Nguyen A. V.: Nucleobases modified azo-polysiloxanes, materials with potential application in biomolecules nanomanipulation. Journal of Polymer Science Part A: Polymer Chemistry, 45, 4240-4248 (2007). DOI: $10.1002 /$ pola.22165

[30] Enea R., Resmerită A-M., Petraru L., Grigoraş C., Scutaru D., Simionescu C. I., Hurduc N.: Synthesis and photochromic behavior of some azo-polysiloxanes modified with nucleobases or donor-acceptor groups. Central European Journal of Chemistry, 5, 981-995 (2007).

DOI: $10.2478 / \mathrm{s} 11532-007-0042-8$

[31] Bian S., Li L., Kumar J., Kim D. Y., Williams J., Tripathy S. K.: Single laser beam-induced surface deformation on azobenzene polymer films. Applied Physics Letters, 73, 1817-1819 (1998). DOI: $10.1063 / 1.122292$

[32] Resmerita A-M., Epure L., Hurduc N., Adès D., Siove A.: Surface properties, thermal behavior, and molecular simulations of azo-polysiloxanes under light stimuli. Insight into the relaxation. Macromolecular Research, 8, 721-729 (2010). DOI: $10.1007 / \mathrm{s} 13233-010-0806-4$

[33] Holme N. C. R., Nikolova L., Ramanujam P. S., Hvilsted S.: An analysis of the anisotropic and topographic gratings in a side-chain liquid crystalline azobenzene polyester. Applied Physics Letters, 70, 1518-1520 (1997).

DOI: $10.1063 / 1.118605$

[34] Lagugné Labarthet F., Buffeteau T., Sourisseau C.: Analyses of the diffraction efficiencies, birefringence, and surface relief gratings on azobenzene-containing polymer films. The Journal of Physical Chemistry B, 102, 2654-2662 (1998).

DOI: $10.1021 / j p 980050 \mathrm{e}$

[35] Sobolewska A., Miniewicz A.: Analysis of the kinetics of diffraction efficiency during the holographic grating recording in azobenzene functionalized polymers. The Journal of Physical Chemistry B, 111, 1536-1544 (2007).

DOI: 10.1021/jp0670211 